\title{
Laparoscopic Natural Orifice Specimen Extraction Surgery versus Conventional Surgery in Colorectal Cancer: A Meta-Analysis of Randomized Controlled Trials
}

\author{
Zhuqing Zhou, Lin Chen, Jie Liu, Fang Ji, Yuanyuan Shang, Xudong Yang, Yao Yang $\mathbb{D}$, \\ and Chuangang Fu $\mathbb{1}$
}

Department of Colorectal Surgery, Department of General Surgery, Shanghai East Hospital, Tongji University School of Medicine, 150 Jimo Road, Shanghai 200120, China

Correspondence should be addressed to Yao Yang; malficeyang@163.com and Chuangang Fu; fugang416@126.com

Received 27 November 2020; Accepted 13 December 2021; Published 18 January 2022

Academic Editor: Vincenzo Pilone

Copyright ( 2022 Zhuqing Zhou et al. This is an open access article distributed under the Creative Commons Attribution License, which permits unrestricted use, distribution, and reproduction in any medium, provided the original work is properly cited.

\begin{abstract}
Objective. This study was to quantitatively synthesize data in randomized controlled trials (RCTs) of laparoscopic resection comparing natural orifice specimen extraction (NOSE) versus conventional laparoscopy (CL) in colorectal cancer. Methods. We identified eligible RCTs by searching seven electronic databases (PubMed, Cochrane Library, Embase, Web of Science, CNKI, CQVIP, Wanfang, and Sinomed). Mean differences (MDs) between groups with 95\% confidence intervals (CIs) were used for continuous outcomes. Event rate ratios (RRs) were also calculated with their 95\% CIs. Results. 1,569 citations were identified from electronic database as of June 2020, and finally, 21 RCTs involving 2,112 patients met the study eligibility criteria and were included. Compared to the CL group, NOSE had longer operation time (MD: $8.14 \mathrm{~min}, 95 \%$ CI: 3.02 to 13.25 , and $p<0.01)$, less estimated blood loss $(-10.64 \mathrm{ml}, 95 \% \mathrm{CI}:-14.92$ to -6.36 , and $p<0.01)$, less hospital stay after surgery $(-2.21$ days, $95 \%$ CI: -3.36 to -1.06 , and $p<0.01)$, shorter time of gas passage after surgery $(-0.58$ days, $95 \%$ CI: -0.82 to -0.34 , and $p<0.01)$, better pain score $(-1.06,95 \% \mathrm{CI}:-3.74$ to -0.37 , and $p<0.01)$, and improved cosmetic scores $(1.93,95 \%$ CI: 0.77 to $3.10, p<0.01)$. Rate ratios of total complications, infection, and incision infection all favored NOSE surgery, with RRs (95\% CIs) of 0.81 (0.71 to 0.93$), 0.34$ (0.21 to 0.54$)$, and 0.24 (0.12 to 0.51$)$, respectively. Conclusion. This report appeared the first comprehensive meta-analysis of RCTs to synthesize data of laparoscopic resection with NOSE versus conventional laparoscopy. NOSE surgery seemed favorable with shorter hospital stay, less pain score, a shorter time to recover along with better cosmetic scores, and less postoperative complications.
\end{abstract}

\section{Introduction}

Colorectal cancer (CRC) remains one of primary causes of cancer-related morbidity and mortality worldwide [1]. As one of the treatment options, laparoscopic surgery has been accepted for decades widely [2]. In recent years, natural orifice specimen extraction surgery (NOSES) is gradually practicing in CRC's treatment and hence causes widespread interests among surgeons [3]. It is reported that NOSE surgery would reduce access trauma in laparoscopic colorectal surgery, with alleviated postoperative pain, faster patient recovery, and a favorable long-term outcome regarding cosmesis and incisional hernia rate [4]. However, a NOSE surgery guideline with adequate evidence has not been formulated to date yet. There were also negative arguments that NOSE surgery may be a risk factor of bacterial contamination of the peritoneal cavity [5]. Nevertheless, relevant studies on NOSE are increasing year by year while few metaanalyses, especially of randomized controlled trials (RCTs), have been carried out. As a result, this topic is still at the level of insufficient evidence $[4,6]$. Given these, we carried out this meta-analysis study of RCTs in a hope to summarize laparoscopic resection data comparing NOSE versus conventional laparoscopy in colorectal cancer. 

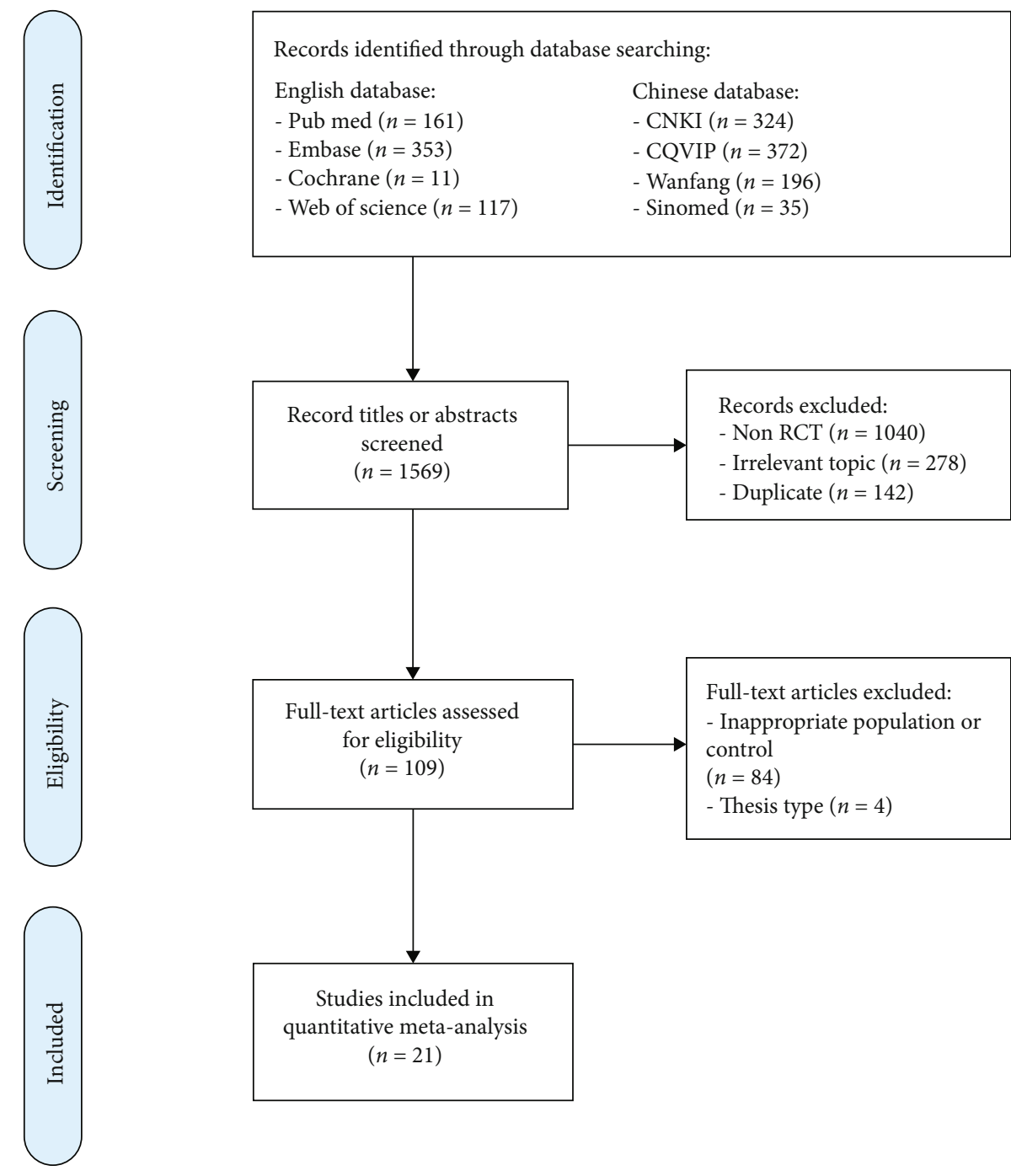

FIGURE 1: Flow diagram of the study search and selection process.

\section{Methods}

2.1. Study Search. We identified eligible RCTs by searching seven electronic databases (PubMed, Cochrane Library, Embase, Web of Science, CNKI, CQVIP, Wanfang, and Sinomed) by using the following terms: "colorectal disease" or "colorectal cancer" or "colorectal tumor" or "colorectal carcinoma" or "colorectal neoplasm" or "rectal disease" or "rectal cancer" or "rectal tumor" or "rectal carcinoma" or "rectal neoplasm" and "natural orifice specimen extraction surgery" or "natural orifice transluminal extraction surgery" or "transrectal specimen extraction" or "transrectal specimen extraction" or "transvaginal specimen extraction" or "no auxiliary incision" or "without auxiliary incision" or "NOSES" or "natural orifice transluminal endoscopic surgery (NOTES)". Additionally, the references of relevant studies on the same topic were manually searched further.

2.2. Study Selection. All studies were carefully assessed for their appropriateness using the study entry criteria as follows: (1) published as original article of RCTs, (2) reported a diagnosis of colorectal cancer as study disease and compared the laparoscopic resection with NOSE versus conventional laparoscopic surgery, and (3) the report language was Chinese or English. If more than one article reported data from the same study, the most recent and complete articles were included. However, those studies without any valid information on resection outcomes were removed.

2.3. Data Extraction and Quality Assessment. In this metaanalysis between laparoscopic resection with NOSE surgery (NOSE group) and conventional laparoscopy (CL group), the following data were extracted from each eligible individual study: (1) the name of first author; (2) year of publication; (3) study groups and number of patients; (4) baseline characteristics such as age and sex; and (5) resection outcomes including operation time, estimated blood loss, gas passage after surgery, various complications, and duration of hospital stay.

Two investigators utilized a uniform structured extraction sheet to extract data from included RCTs. If any disagreement was noted, a third investigator was asked to 


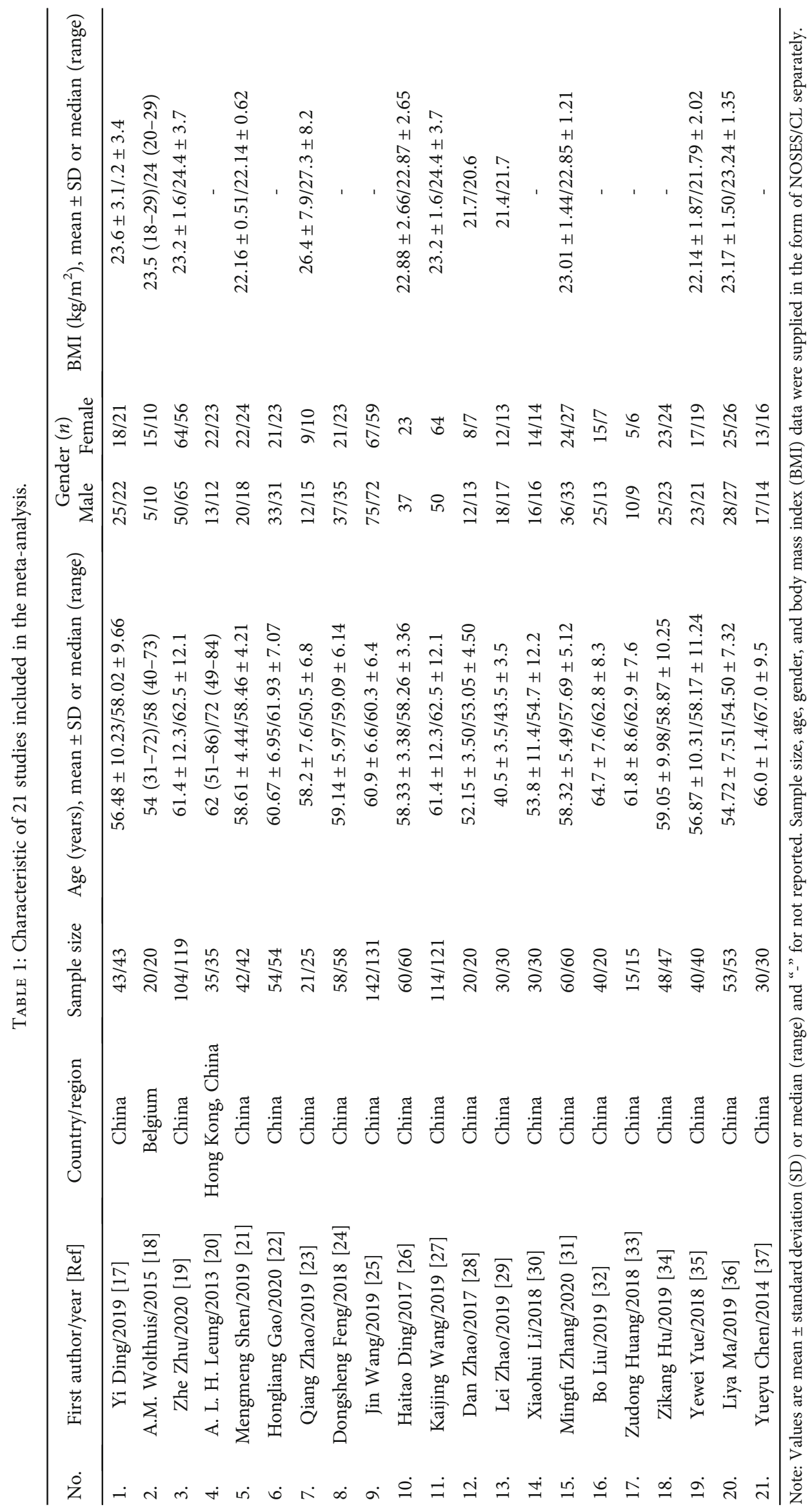




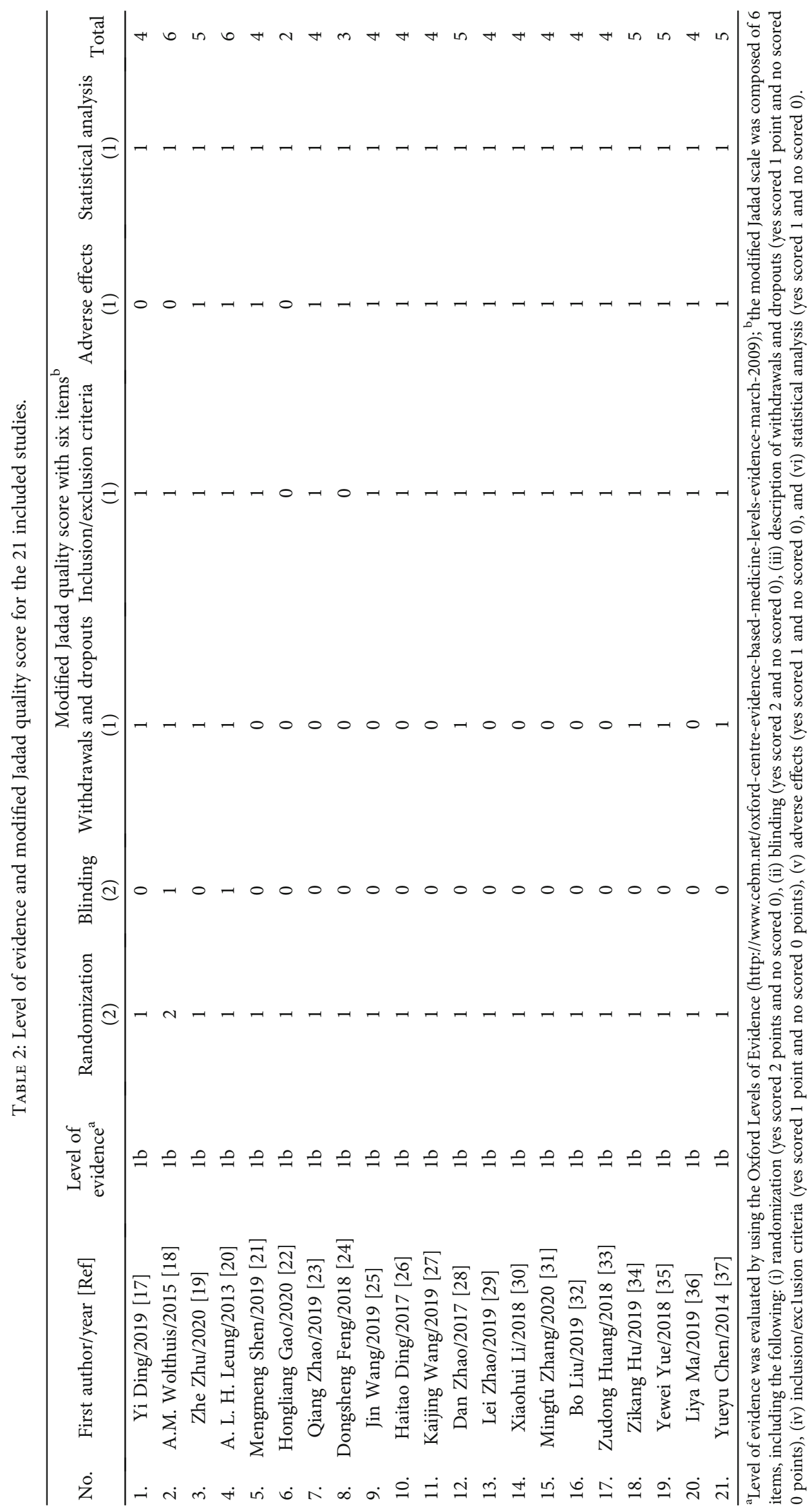




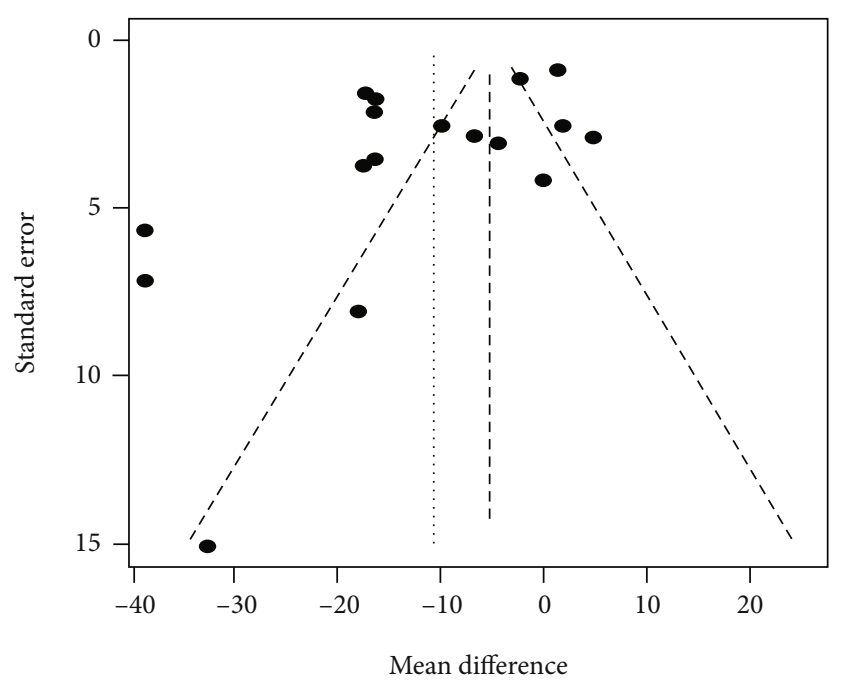

(a) Blood loss (ml)

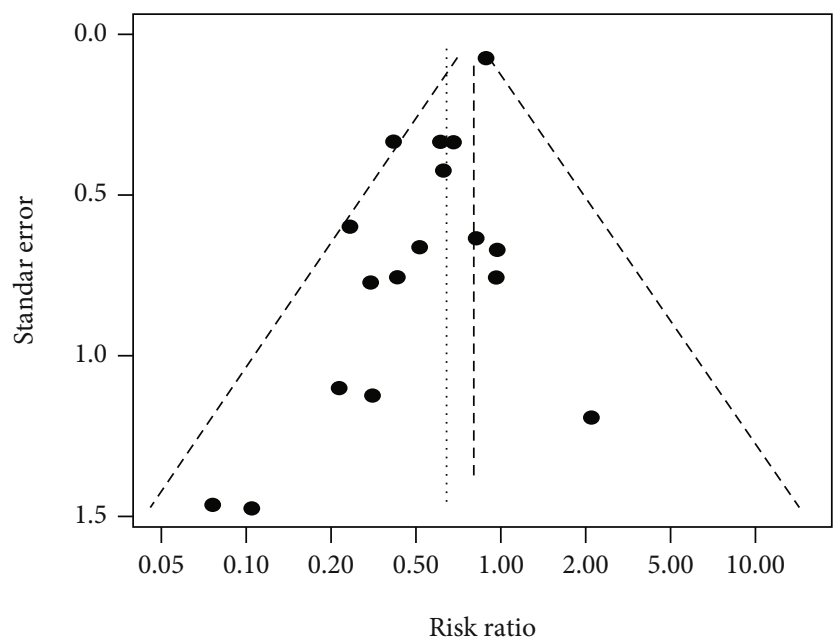

(c) Total postoperative complications

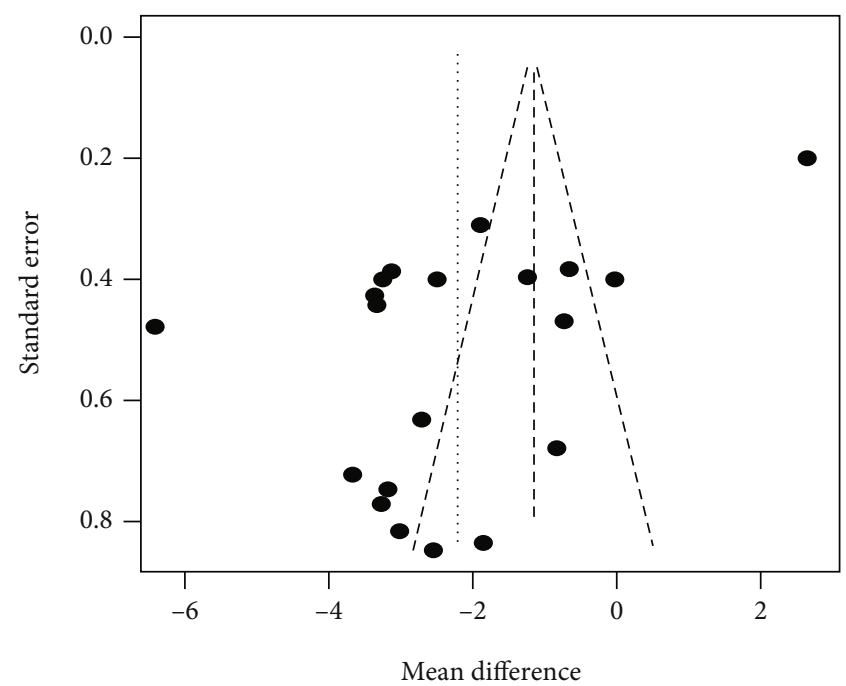

(b) Hospital stay after surgery (days)

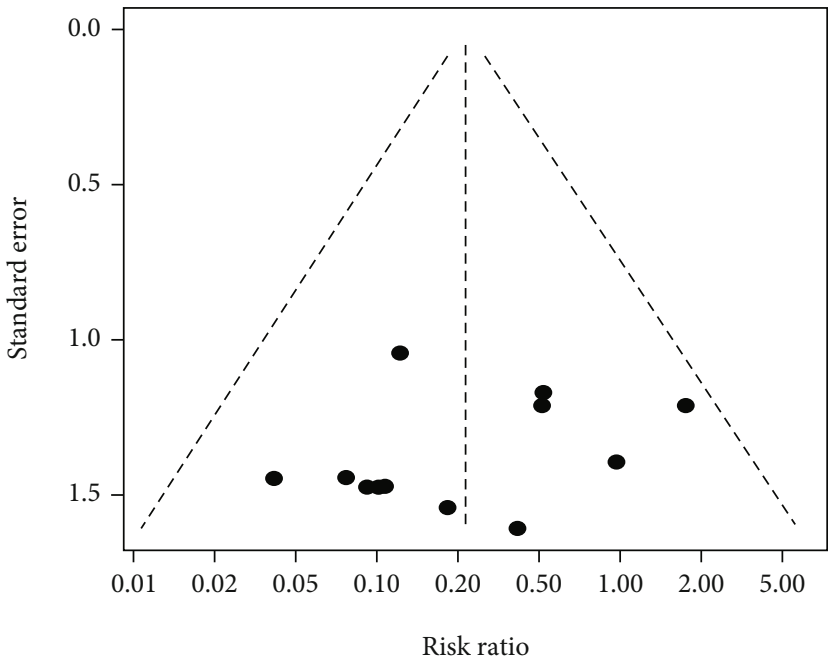

(d) Incision infection

FIgURe 2: Funnel plot of the meta-analysis using the rate ratios against their standard errors. (a) Estimated blood loss in millilitres; (b) hospital stay after surgery in days; (c) total postoperative complications; (d) incision infection.

reach a final agreement. The potential risk of study bias was assessed according to the preferred reporting items for systematic reviews and meta-analysis recommendations [7]. The level of evidence was evaluated by using the Oxford Levels of Evidence $[8,9]$. Study quality was assessed by using the modified Jadad scale, which involves six items to evaluate the methodological quality of RCTs [10-12]. Its score range was 0 to 8 , with a higher score showing better report quality. In this study, a score of 1 to 3 indicated low quality and 4 to 8 for high quality.

2.4. Statistical Analysis. We used R 3.4.4 (R Foundation for Statistical Computing, Vienna, Austria; http://www.Rproject.org/) and the Meta package [13] for this metaanalysis. For continuous outcome data, mean differences (MDs) along with their 95\% confidence intervals (CIs) were used as their main effect measures. When the mean and standard deviation were not provided directly, we estimated them from the median, range, and size of the study samples [14]. For binary event data, the rate ratios (RRs) were calculated with $95 \%$ CIs. Heterogeneity was defined as an $I^{2}$ value of more than 50\% [15] or $p$ value of less than 0.10 from Cochrane $Q$ test [16]. These two statistics evaluate the percentage of variability attributable to study heterogeneity instead of by chance. Therefore, when an outcome measure showed negligible heterogeneity, we used a fixed-effect model for its data pooling instead of random-effects model. The funnel plots were visually inspected for the measures of most included RCTs being conducted to statistically evaluate publication bias [15]. For any statistical test, significance was defined as a two-tailed $p$ value of 0.05 or less.

\section{Results}

3.1. Search Results and Study Characteristics. Initially, 1,569 citations were identified from electronic database as of June 


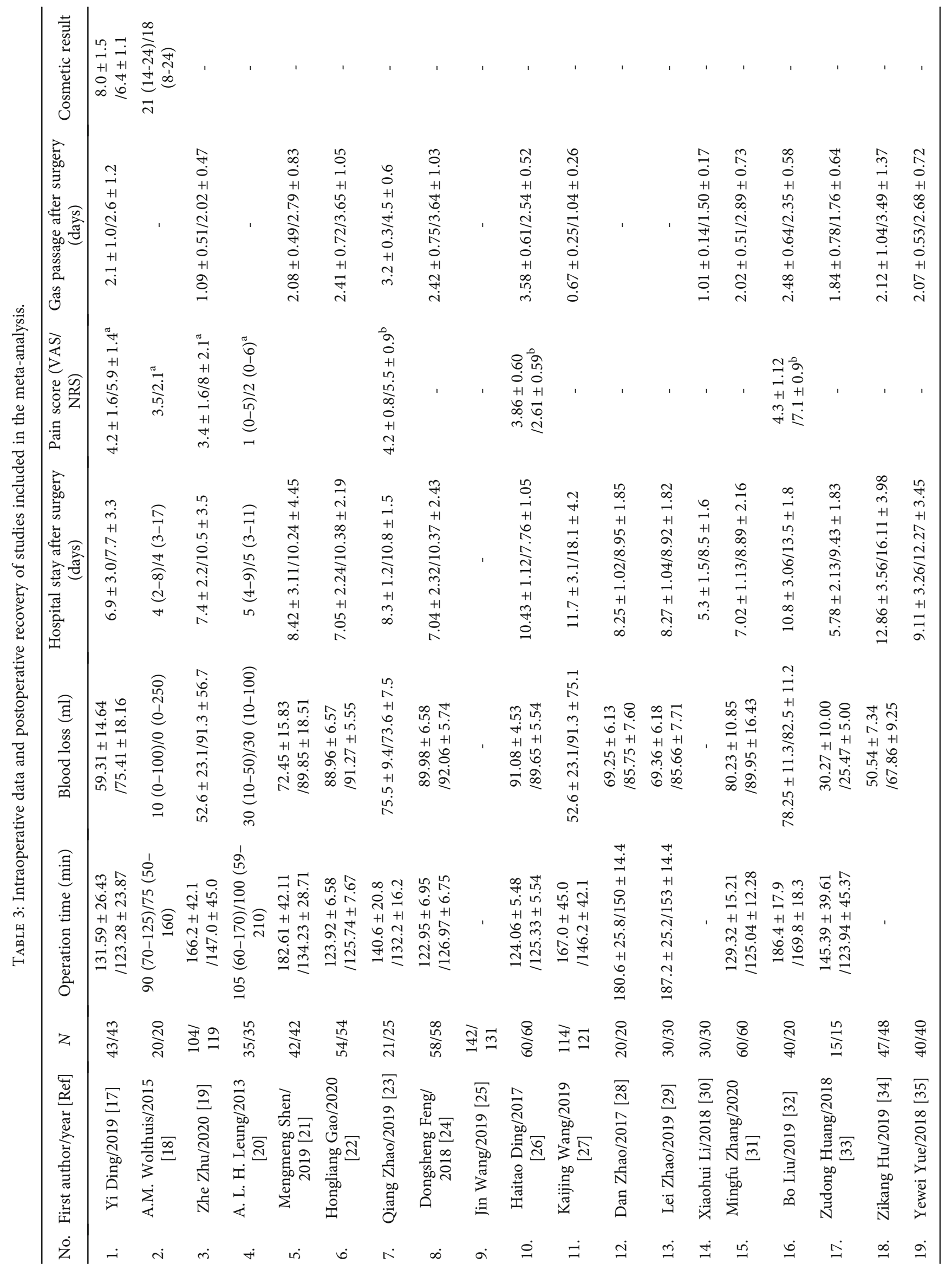




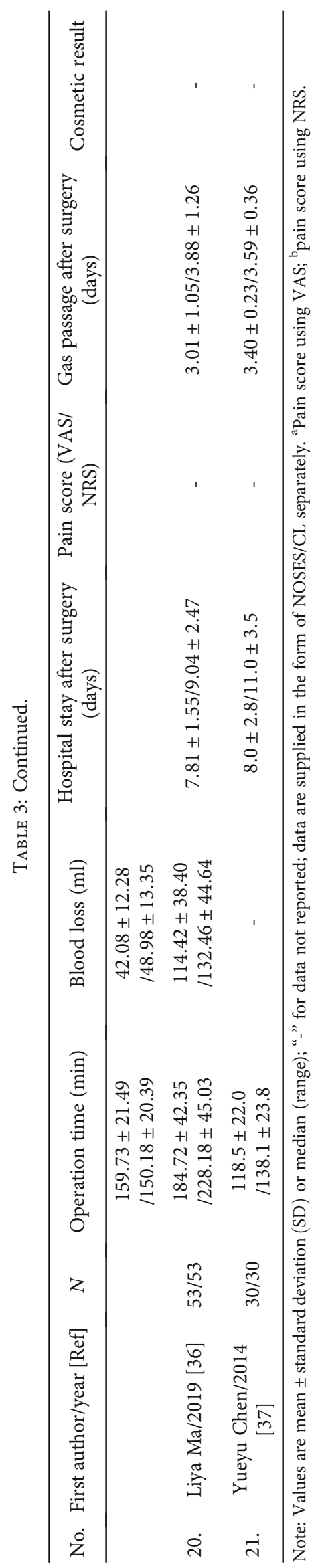


NOSES

\begin{tabular}{|c|c|c|c|c|c|}
\hline \multirow[b]{2}{*}{ Study } & \multicolumn{5}{|c|}{ NOSES } \\
\hline & Total & Mean & \multicolumn{2}{|c|}{ SD Total } & Mean \\
\hline Yi ding 2019 & 43 & 131.59 & 26.43 & 43 & 123.28 \\
\hline A.M.wolthuis 2015 & 20 & 93.75 & 13.75 & 20 & 90.00 \\
\hline Zhe zhu 2020 & 104 & 166.20 & 42.10 & 119 & 147.00 \\
\hline A. L. H. leung 2013 & 35 & 105.00 & 27.50 & 35 & 100.00 \\
\hline Mengmeng shen 2019 & 42 & 182.61 & 42.11 & 42 & 134.23 \\
\hline Hongliang gao 2020 & 54 & 123.92 & 6.58 & 54 & 125.74 \\
\hline Qiang zhao 2019 & 21 & 140.60 & 20.80 & 25 & 132.20 \\
\hline Dongsheng feng 2018 & 58 & 122.95 & 6.95 & 58 & 126.97 \\
\hline Haitao ding 2017 & 60 & 124.06 & 5.48 & 60 & 125.33 \\
\hline Kaijing wang 2019 & 114 & 167.00 & 45.00 & 121 & 146.20 \\
\hline Dan zhao 2017 & 20 & 180.60 & 25.80 & 20 & 150.00 \\
\hline Lei zhao 2019 & 30 & 187.20 & 25.20 & 30 & 153.00 \\
\hline Mingfu zhang 2020 & 60 & 129.32 & 15.21 & 60 & 125.04 \\
\hline Bo liu 2019 & 40 & 186.40 & 17.90 & 20 & 169.80 \\
\hline Zudong huang 2018 & 15 & 145.39 & 39.61 & 15 & 123.94 \\
\hline Yewei yue 2018 & 40 & 159.73 & 21.49 & 40 & 150.18 \\
\hline Liya ma 2019 & 53 & 184.72 & 42.35 & 53 & 228.18 \\
\hline Yueyu chen 2014 & 30 & 118.50 & 22.00 & 30 & 138.10 \\
\hline Random effects model & 839 & & & 845 & \\
\hline \multicolumn{6}{|c|}{ Heterogeneity: $I^{2}=92 \%, \tau^{2}=90.4, p<0.01$} \\
\hline \multicolumn{6}{|c|}{ Test for overall effect: $z=3.12(p<0.01)$} \\
\hline
\end{tabular}

CL

$\mathrm{SD}$

23.87

27.50

45.00

37.75

28.71

7.67

16.20

6.75

5.54

42.10

14.40

14.40

12.28

18.30

45.37

20.39

45.03

23.80

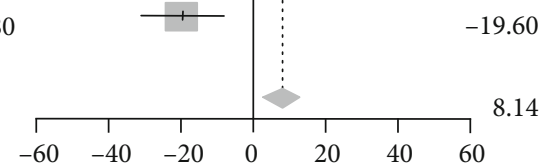

Favours NOSES Favours CL

(a) Operation time (min)

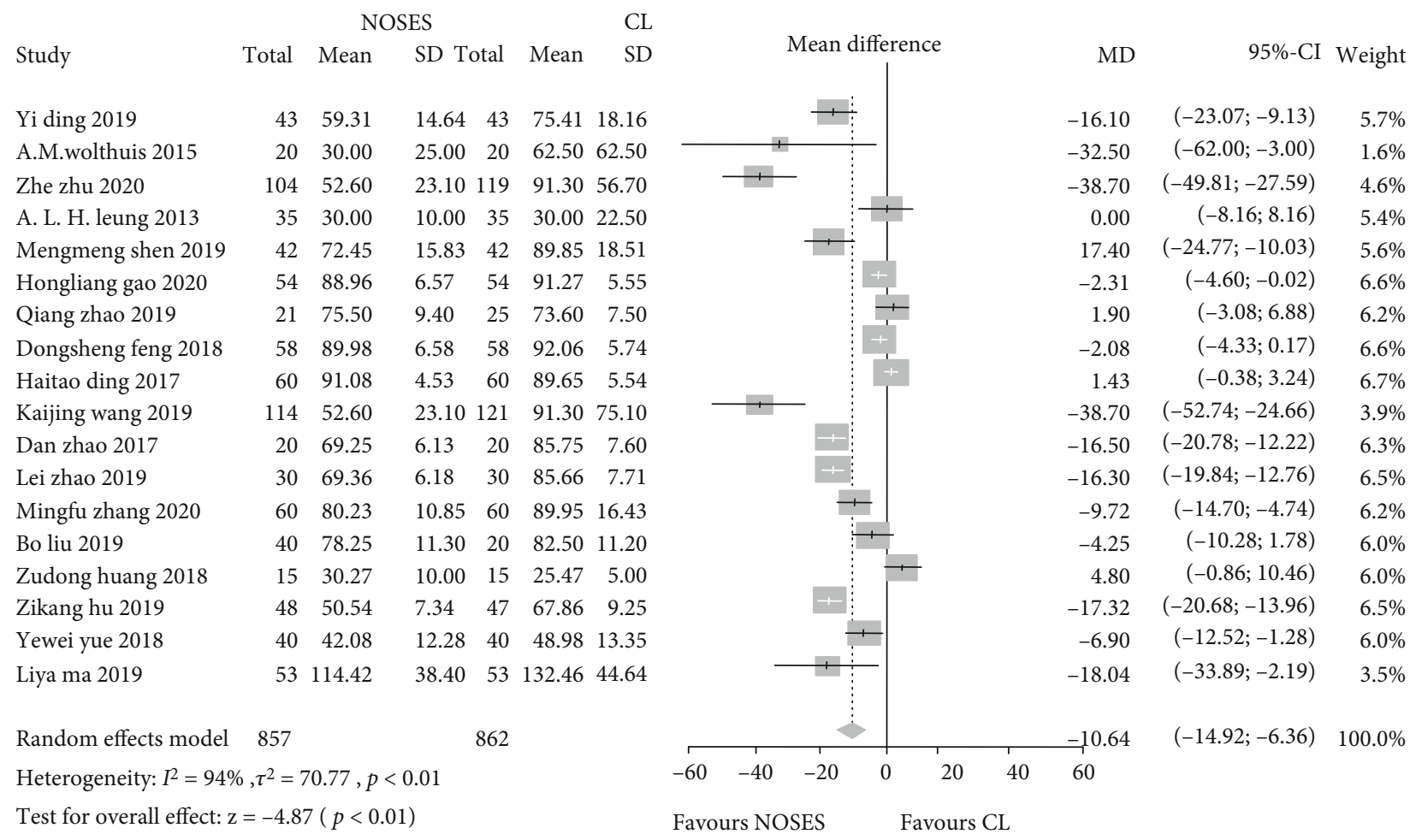

(b) Blood loss (ml)

Figure 3: Continued. 


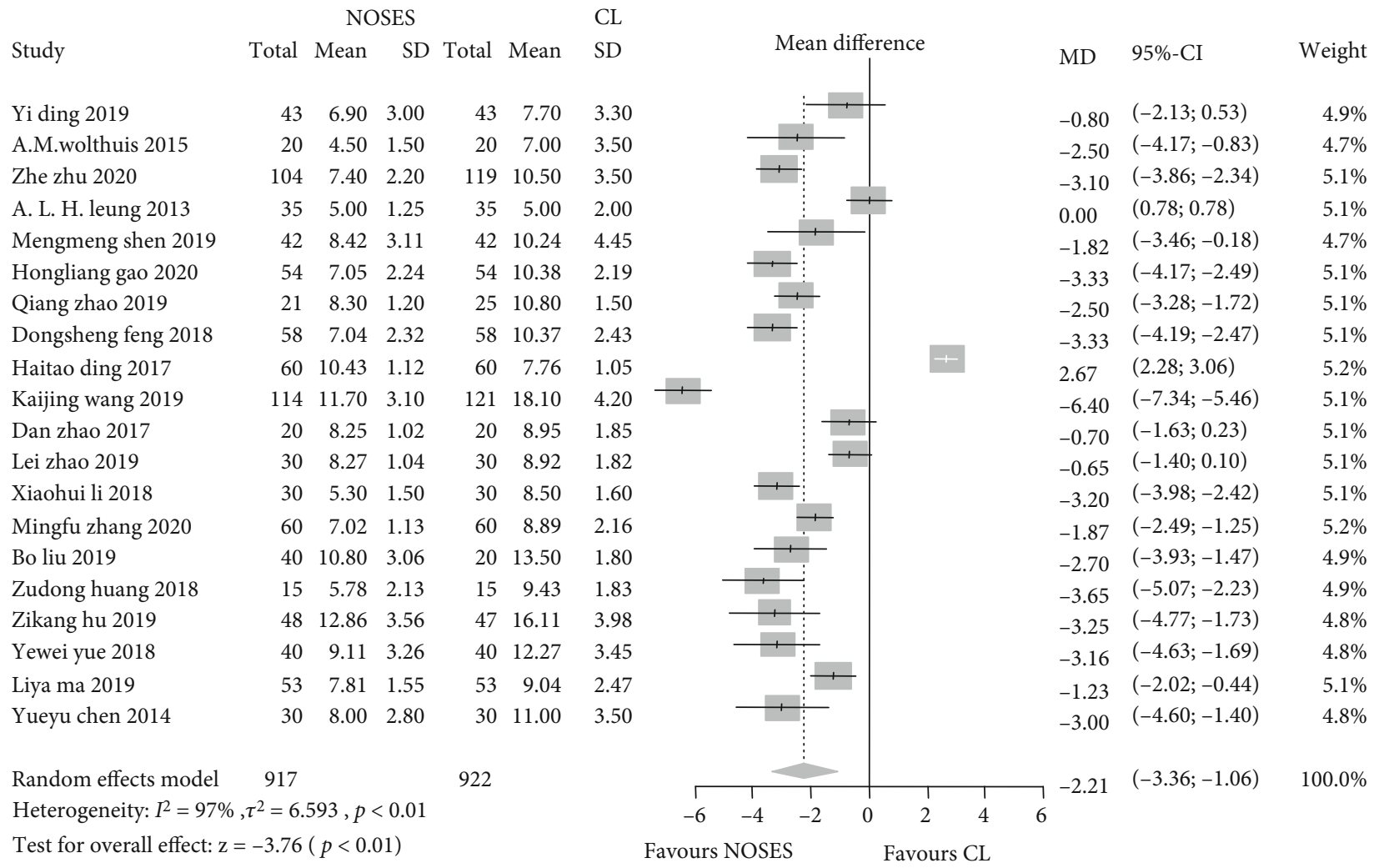

(c) Hospital stay after surgery (days)

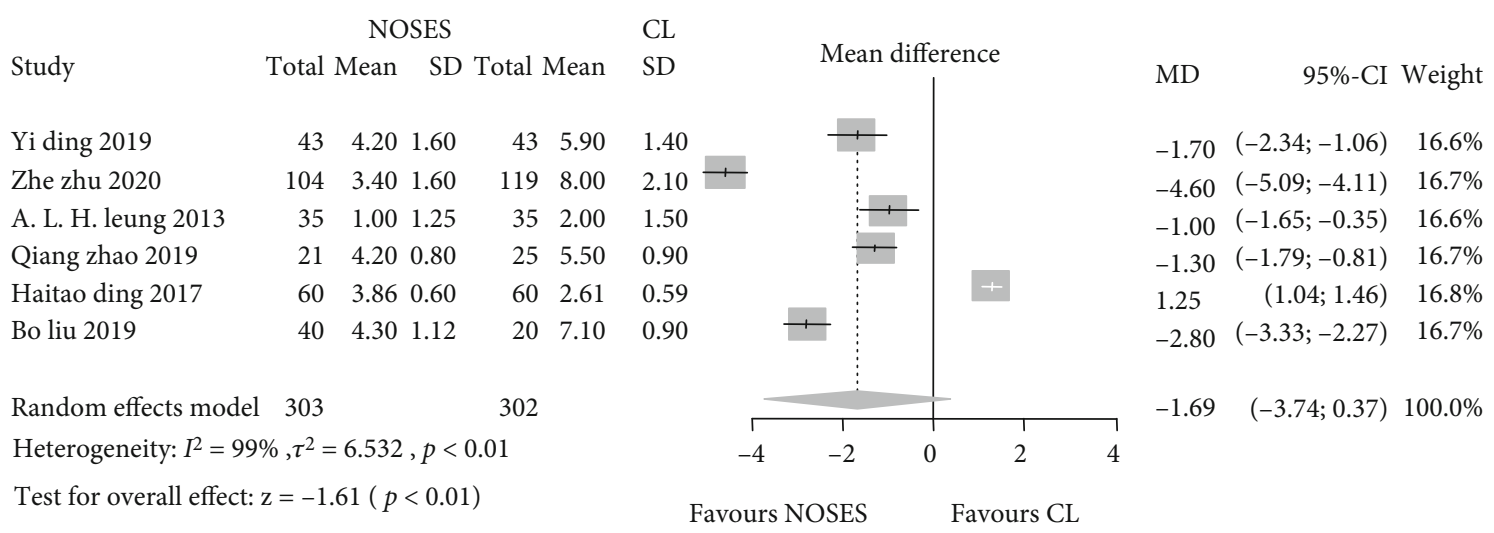

(d) Pain score (VAS/NRS)

Figure 3: Continued. 


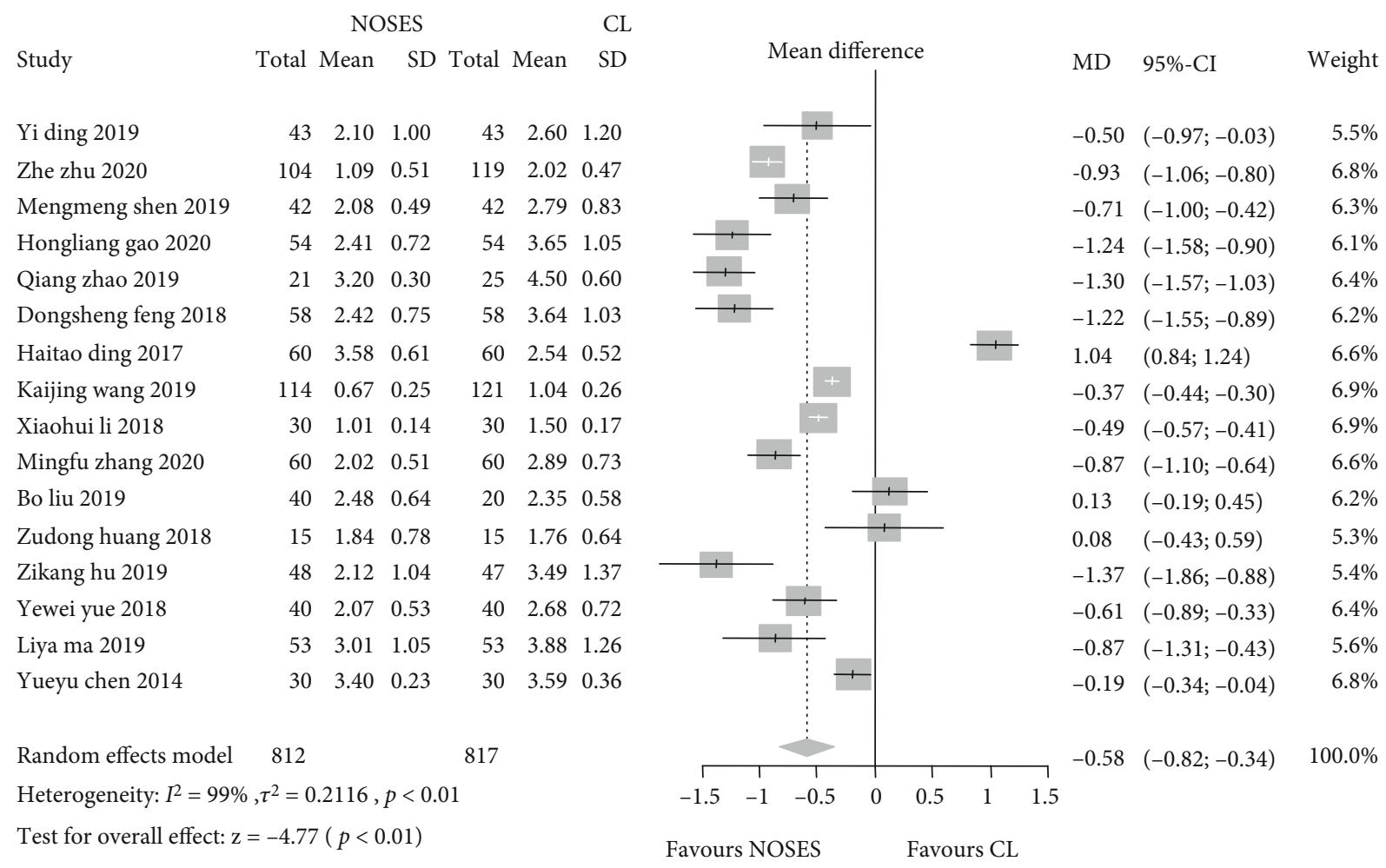

(e) Gas passage after surgery (days)

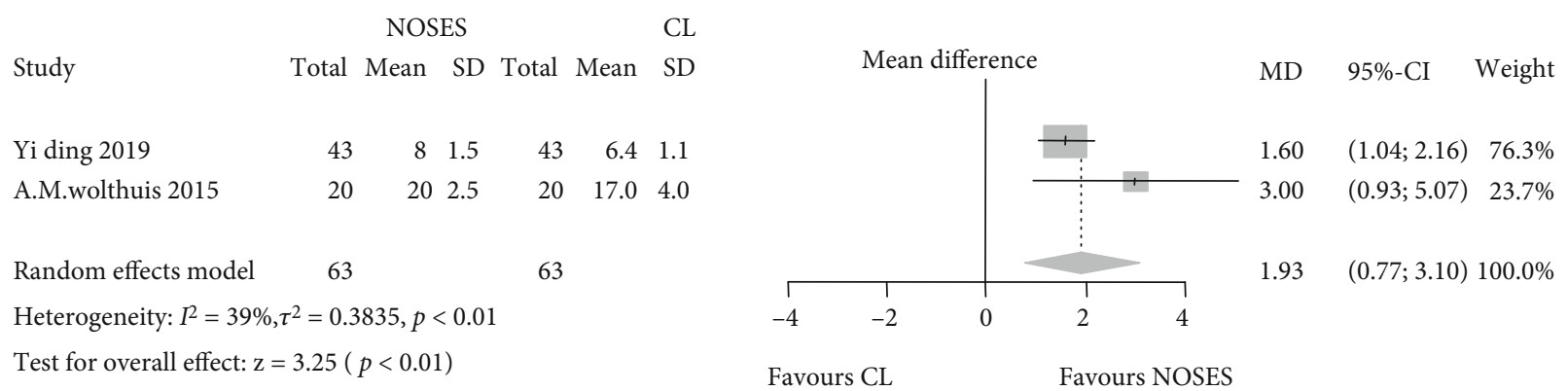

(f) Cosmetic result

FIgURE 3: Forest plots of intraoperative data and postoperative recovery between the NOSE group and the CL group. (a) Operation time in minutes; (b) estimated blood loss in millilitres; (c) hospital stay after surgery in days; (d) pain score; (e) gas passage after surgery in days; (f) cosmetic result.

2020 (cut-off date), of which 1,460 were excluded for a variety of reasons after the screening of citation titles and abstracts, leaving 109 studies for further full-text assessment. Of them, 88 studies were excluded due to their inappropriate study population or thesis type. Finally, a total of 21 RCTs [17-37] involving 2,112 patients met the study eligibility criteria and were included (Figure 1).

Only patients from the NOSE group or the CL group according to laparoscopic resection methods were included in our meta-analysis. Four studies [38-41] published as thesis and not in peer-reviewed journals were excluded. One study [42] with a printing error but was repaired and one study [43] in Russian were excluded. For four studies with more than two arms, we removed the open surgery group from two studies $[19,27]$ and the laparoscopic surgery plus a traditional nursing group [30] or combined two NOSEStype arms into one [32]. The main study characteristics are shown in Table 1.

3.2. Study Quality and Publication Bias. The results of quality assessment by the modified Jadad scale were as follows: two articles scored 6, five scored 5, twelve scored 4, one scored 3, and one scored 2. In summary, 19 out of 21 studies earned a score of 4 or more. All of the 21 articles were on RCT design and met $1 \mathrm{~b}$ level of evidence. These generally suggested their high study quality (Table 2).

The funnel plots were drawn for effect outcomes of estimated blood loss, hospital stay after surgery, total postoperative complications, and incision infection (Figure 2). Incision infection showed some symmetry, and no 


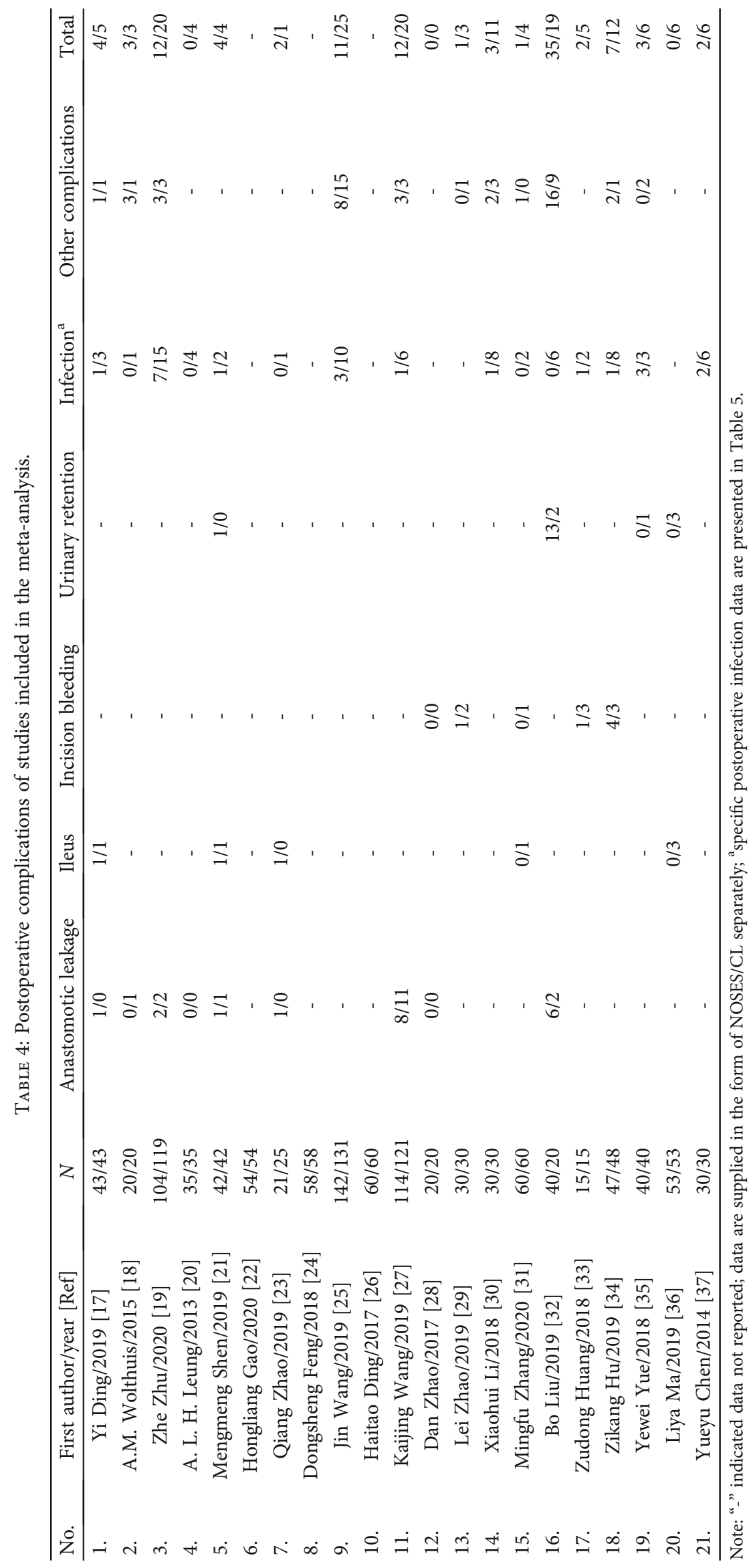




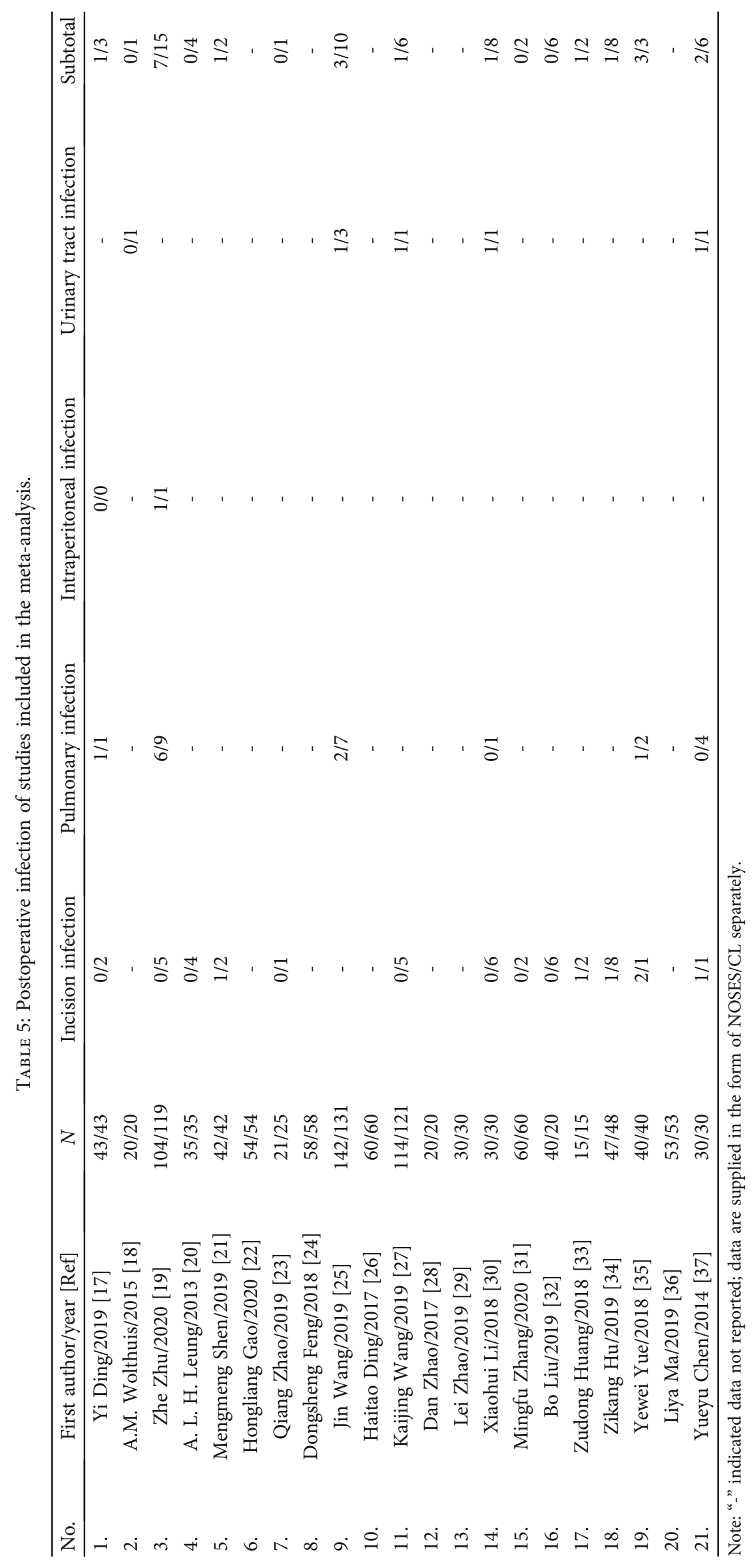


CL

Author (s) and year Incision bleeding Total Incision bleeding Total

Dan zhao 2017

Lei zhao 2019

Mingfu zhang 2020

Zudong huang 2018

Zikang hu 2019

Fixed effect model

Random effects model

Heterogeneity: $I^{2}=0 \%, \tau^{2}=0, p=0.6679$

Test for overall effect (fixed effect): $p=0.5205$

Test for overall effect (random effects): $p=0.5205$

\section{tal}

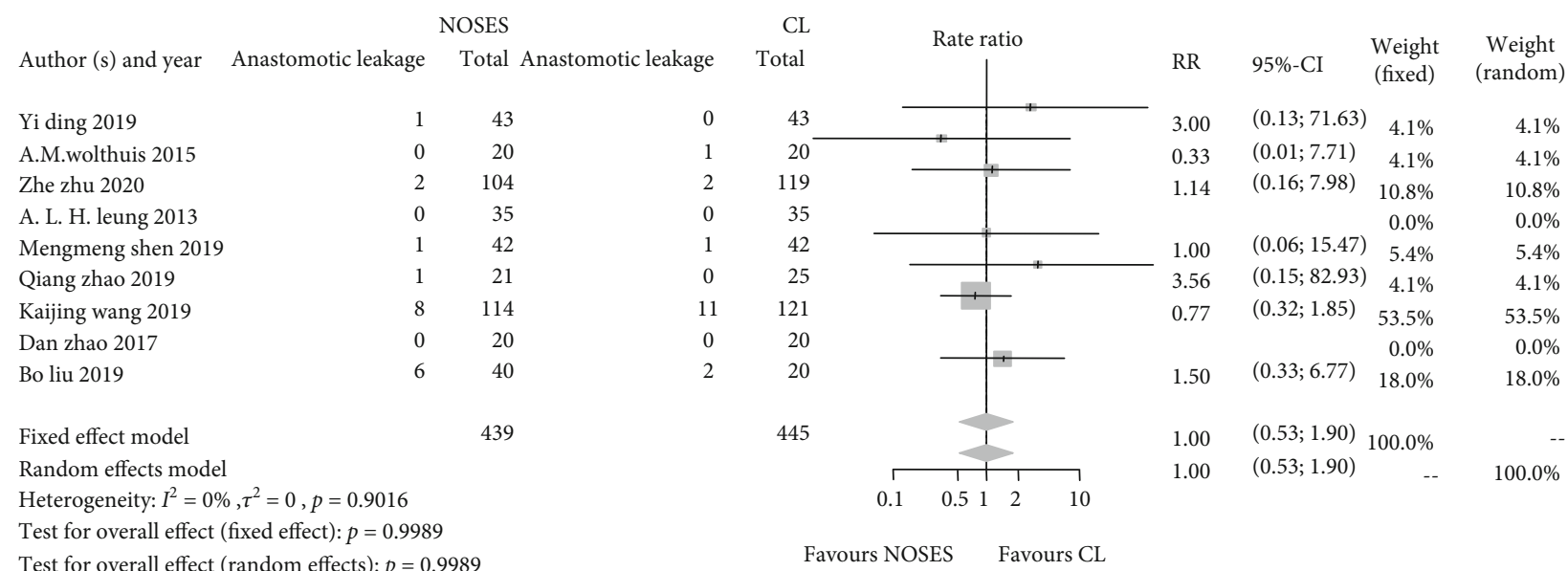

(a) Anastomotic leakage

\section{NOSES CL}

Author (s) and year Ileus Total Ileus Total

$\begin{array}{lllll}\text { Yi ding 2019 } & 1 & 43 & 1 & 43 \\ \text { Mengmeng shen 2019 } & 1 & 42 & 1 & 42 \\ \text { Qiang zhao 2019 } & 1 & 21 & 0 & 25 \\ \text { Mingfu zhang 2020 } & 0 & 60 & 1 & 60 \\ \text { Liya ma 2019 } & 0 & 53 & 3 & 53\end{array}$

Fixed effect model 219

Random effects model

Heterogeneity: $I^{2}=0 \%, \tau^{2}=0, p=0.6470$

Test for overall effect (fixed effect): $p=0.5969$

Test for overall effect (random effects): $p=0.5969$

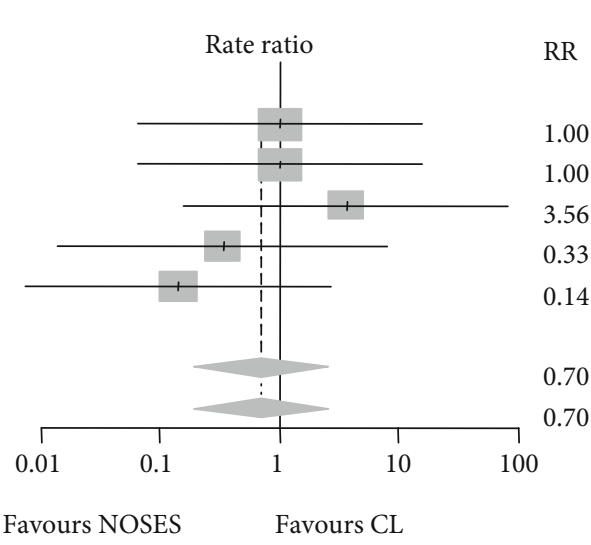

(b) Ileus

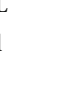

Weight Weight 95\%-CI (fixed) (random)

$(0.06 ; 15.48) \quad 22.9 \% \quad 22.9 \%$

$(0.06 ; 15.47) \quad 22.9 \% \quad 22.9 \%$

$(0.15 ; 82.93) \quad 17.3 \% \quad 17.3 \%$

$(0.01 ; 8.02) \quad 17.0 \% \quad 17.0 \%$

$(0.19 ; 2.60) \quad 100.0 \%$

$(0.19 ; 2.60) \quad--\quad 100.0 \%$
$(0.01 ; 2.70) \quad 19.9 \% \quad 19.9 \%$ 


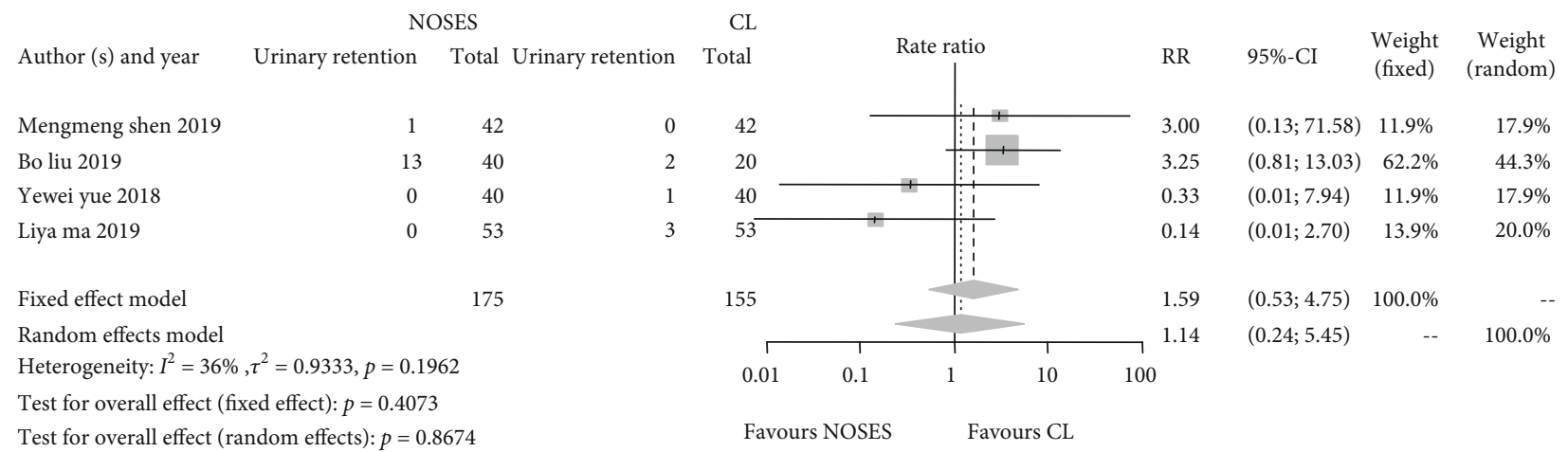

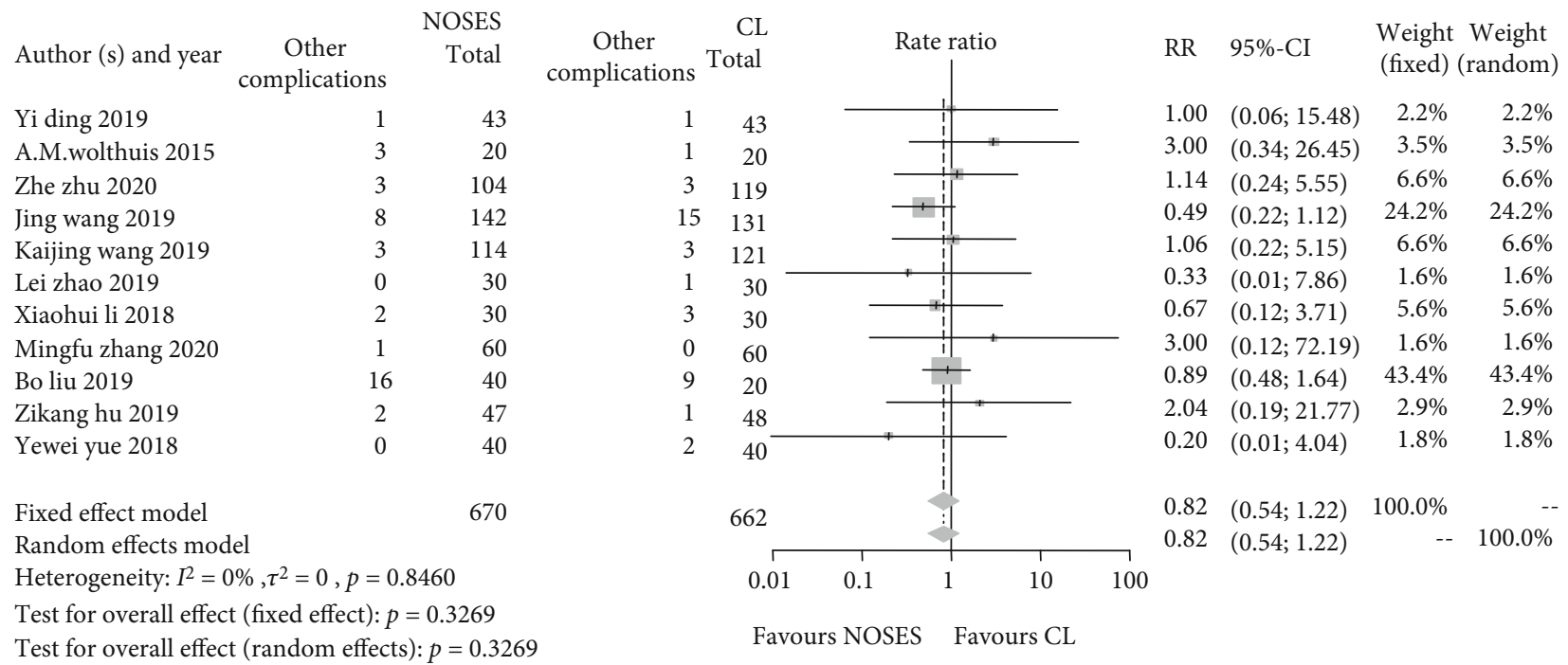

(e) Other complications

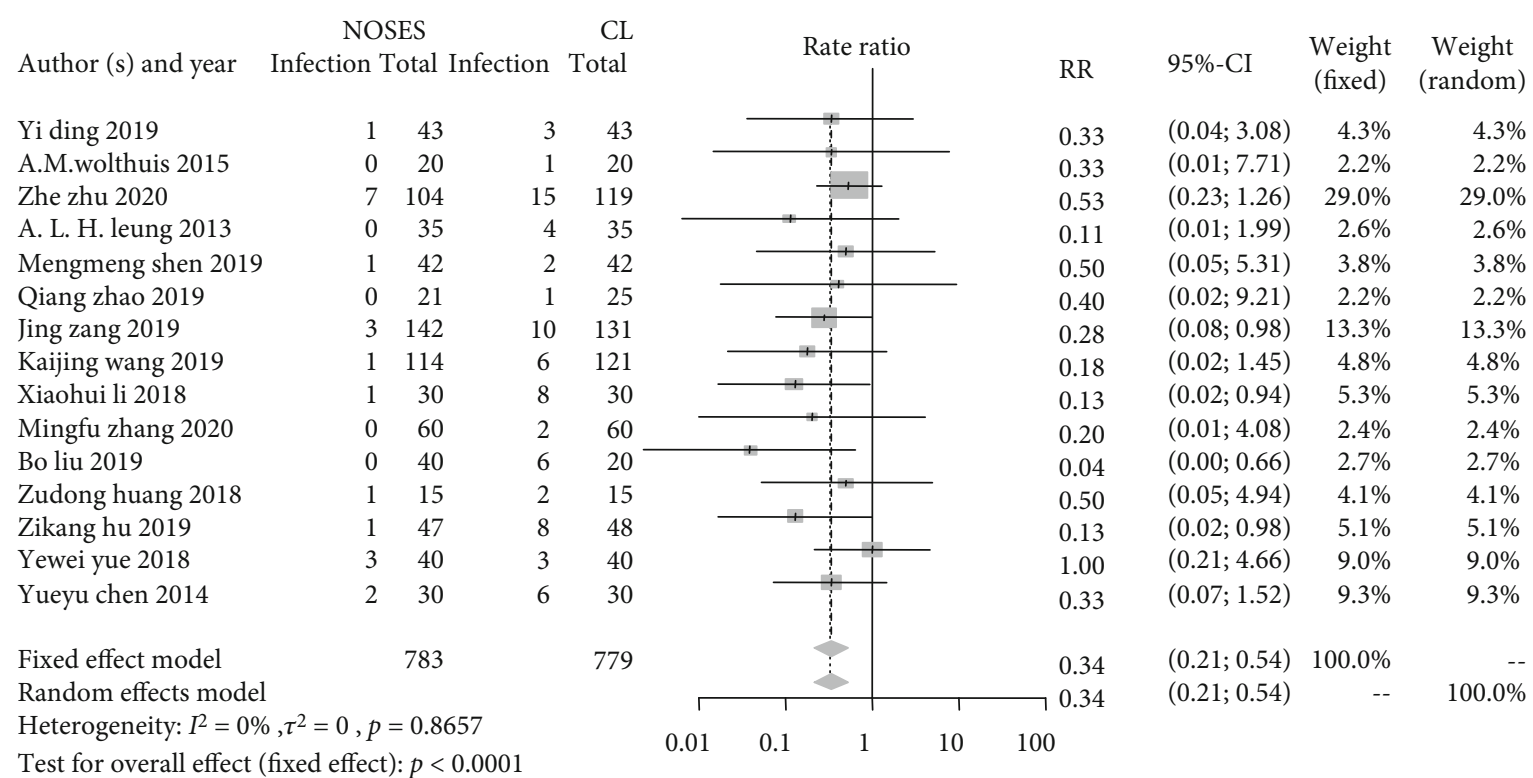

Test for overall effect (random effects): $p<0.0001$

Favours NOSES Favours CL

(f) Infection

Figure 4: Continued. 


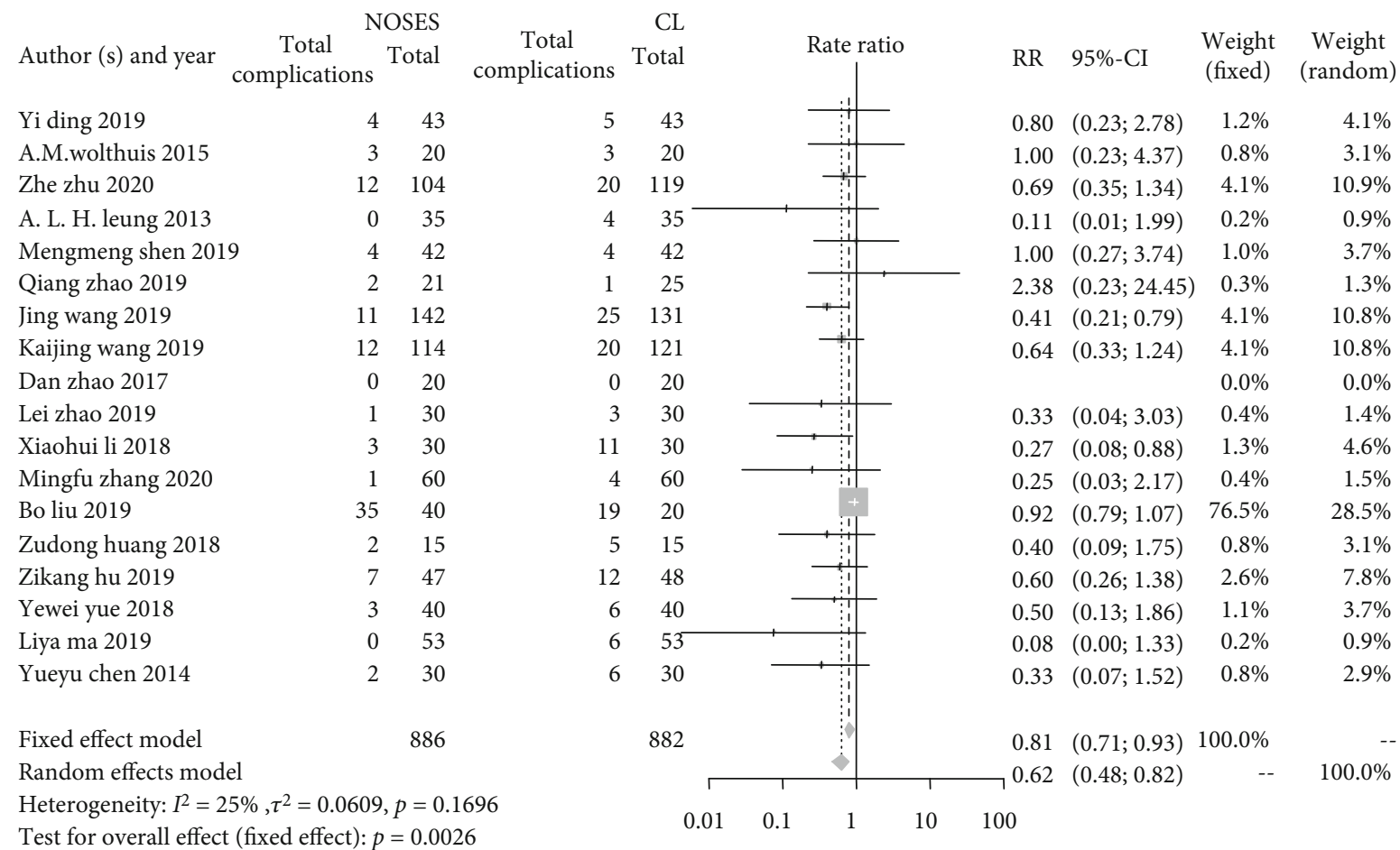

Test for overall effect (random effects): $p=0.0006$

Favours NOSES Favours CL

(g) Total complications

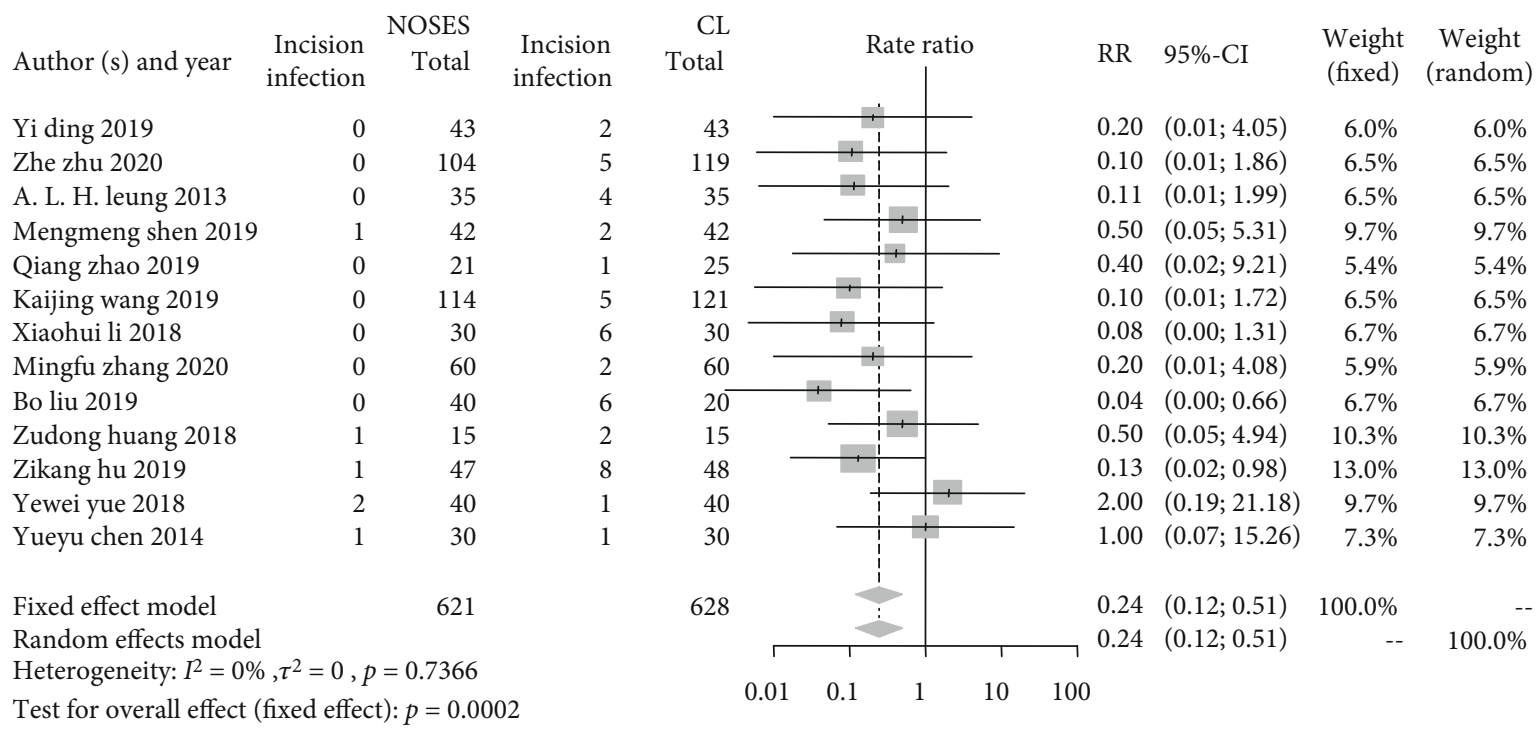

Test for overall effect (random effects): $p=0.0002$

Favours NOSES Favours CL

(h) Incision infection

FIGURE 4: Forest plots of postoperative complications between the NOSE group and the CL group. (a) Anastomotic leakage; (b) ileus; (c) incision bleeding; (d) urinary retention; (e) other complications; (f) infection; (g) total complications; (h) incision infection.

statistically significant publication bias was found $(p=0.3103)$. Funnel plots for the other outcomes showed asymmetry (Figure 2).

\subsection{Meta-Analysis Results}

3.3.1. Intraoperative Data and Postoperative Recovery. The patient intraoperative data and postoperative recovery of the included RCTs are presented in Table 3. Operation time, estimated blood loss, and hospital stay after surgery were reported in 18, 18, and 20 studies, respectively. An 8 minutes of mean operation time was prolonged in the NOSE group as compared to the CL group (MD: $8.14 \mathrm{~min}, 95 \% \mathrm{CI}: 3.02$ to 13.25 , and $p<0.01)$. However, intraoperative estimated blood loss was decreased in the NOSE group as compared to the CL group (MD: $-10.64 \mathrm{ml}, 95 \% \mathrm{CI}:-14.92$ to -6.36 , 
TABLE 6: Recurrence and overall survival of studies included in the meta-analysis.

\begin{tabular}{lccccc}
\hline No. & First author/year [Ref] & Patients, $n$ & Duration of follow-up, months & Recurrence, $n$ & Overall survival, $n$ \\
\hline 1. & Yi Ding/2019 [17] & $43 / 43$ & $(12-45) /(12-45)$ & $3 / 1$ & - \\
2. & Mingfu Zhang/2020 [31] & $60 / 60$ & $(12-24) /(12-24)$ & $12 / 11$ & - \\
3. & Zikang Hu/2019 [34] & $47 / 48$ & $24 / 24$ & $8 / 9$ & $35 / 35$ \\
4. & Yewei Yue/2018 [35] & $40 / 40$ & $24 / 24$ & $0 / 0$ & - \\
5. & Yueyu Chen/2019 [37] & $30 / 30$ & $28(3-48) / 28(3-48)$ & $34 / 30$ \\
\hline
\end{tabular}

Note: -: not reported. Data are supplied in the NOSES/CL form.

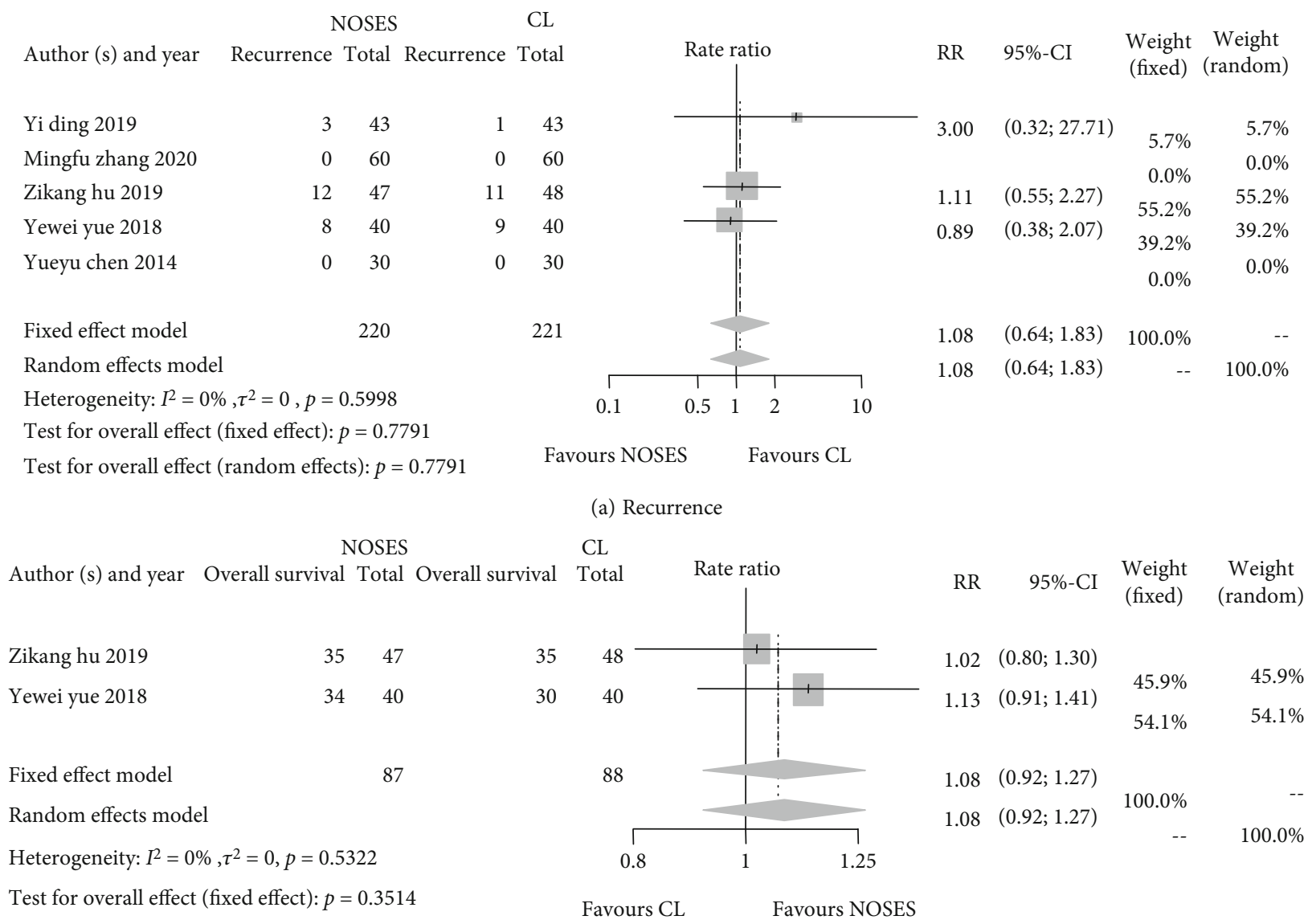

(b) Overall survival

FIGURE 5: Forest plots of recurrence and overall survival rate between the NOSE group and the CL group. (a) Disease recurrence rate; (b) overall survival rate.

and $p<0.01)$. Moreover, hospital stay after surgery was shortened in the NOSE group significantly (MD: -2.21 days, 95\% CI: -3.36 to -1.06 , and $p<0.01)$. Gas passage after surgery was reported in 16 studies and was also shortened in the NOSE group (MD: -0.58 days, $95 \% \mathrm{CI}:-0.82$ to -0.34 , and $p<0.01)$; pain score was improved in the NOSE group (MD: $-1.06,95 \% \mathrm{CI}:-3.74$ to -0.37 , and $p<0.01$ ); cosmetic result seemed better in the NOSE group (MD: 1.93, 95\% CI: 0.77 to 3.10 , and $p<0.01$ ) (see Figure 3 for details).

3.3.2. Postoperative Complications. The postoperative complications of the included RCTs are presented in Table 4, and various postoperative infections are detailed in
Table 5. Postoperative complications were reported in 18 RCTs. 102 out of 886 patients (11.5\%) developed postoperative complications in the NOSE group while 154 out of 882 patients $(17.5 \%)$ in the CL group (RR of 0.81 , $95 \%$ CI 0.71 to 0.93 , and $p=0.003$ in the fixed-effect model, Figure 4). And this improved trend was also shown in the postoperative infection (RR: $0.34,95 \% \mathrm{CI}$ : 0.21 to 0.54 , and $p<0.0001)$, especially in the incision infection (RR: $0.24,95 \% \mathrm{CI}: 0.12$ to 0.51 , and $p=0.0002$ ). However, no significant rate differences were found between the two groups in terms of anastomotic leakage (RR: 1.00, 95\% CI: 0.53 to 1.90 , and $p=0.9989$ ), ileus (RR: $0.70,95 \%$ CI: 0.19 to 2.60 , and $p=0.5969$ ), incision bleeding (RR: $0.72,95 \%$ 
CI: 0.26 to 1.97 , and $p=0.5205$ ), urinary retention (RR: 1.14, $95 \%$ CI: 0.24 to 5.45 , and $p=0.8674)$, and other complications (RR: $0.82,95 \%$ CI: 0.54 to 1.22 , and $p=$ 0.3269 ) (Figure 4).

3.3.3. Recurrence and Overall Survival. Disease recurrent data were reported in five studies and overall survival in two studies (Table 6). No significant differences for both survival-related outcomes were found between the two groups: RR of $1.08,95 \%$ CI 0.64 to 1.83 , and $p=0.7791$ for event recurrence rate and $1.08,95 \%$ CI 0.92 to 1.27 , and $p$ $=0.3514$ for overall survival rate (Figure 5).

\section{Discussion}

To our knowledge, this report appeared the first comprehensive meta-analysis to synthesize RCT data regarding NOSE versus traditional laparoscopic colorectal cancer surgery. The large-sized meta-analysis of 21 RCTs demonstrated that laparoscopic resection with NOSE surgery reduced intraoperative estimated blood loss, relieved postoperative pain, accelerated postoperative recovery, and decreased the incidence of postoperative complications as well.

The terminology regarding NOSE surgery means that the surgical specimen resection is conducted intra-abdominally, and then, the specimen is taken out by opening a hollow organ such as anus, vagina, or mouth to communicate with the outside of the body [44]. Laparoscopic surgery combined with NOSE avoids incisions on the abdominal wall and reduces pain and wound complications, along with a shorter recovery time, etc. [45]. Besides, there was no auxiliary incision on the abdominal wall, and only a few small puncturing scars remained, indicating an excellent minimally invasive effect [46].

Given these reasons above, it was expected that NOSE surgery showed a better prognosis in terms of intraoperative data, postoperative recovery, and complications. NOSE surgery had less estimated blood loss (approximately $11 \mathrm{ml}$ ), and it may be due to no auxiliary incision, reducing the amount of wound bleeding. In the meantime, these results suggested that patients in NOSE group had less postoperative pain, faster recovery than the CL group, which also might be due to no auxiliary incision. The incidence of postoperative complications is an important indicator to evaluate the feasibility of NOSES. The total postoperative complication results suggested a significantly lower risk of complications ( $\mathrm{RR}=0.62,95 \%$ CI 0.48 to 0.82 , and $p=0.0006$ ), especially in the incision infection. Therefore, in recent years, great advances in NOSES lead to a new tendency in CRC's surgical therapy in China and even other countries around the world. Given these, "Expert consensus of natural orifice specimen extraction surgery in colorectal neoplasm (2019)" and "International consensus on NOSES for colorectal cancer (2019)" were published along with individual reports $[44,46]$.

On the other hand, however, NOSE surgery had a slightly longer mean operation time (8 minutes) as compared to the CL group. The reasons behind it may include
(1) the operation space inside the natural cavity is narrow so that the anastomosis is more time-consuming and (2) surgical proficiency of the surgeon with a possible learning curve. Beginners require a learning process to perform this new type of surgery. As for disease recurrence and overall survival rate, there was no significant difference noted between the NOSE group and the CL group, suggesting that there was likely no significant difference in the long-term efficacy. For postoperative complications, new studies with adequate sample size may be also needed to differentiate them later in the future. Even so, laparoscopic NOSES was, to some extent, a safe extraction method for colorectal diseases.

There were several limitations in this report. First, the meta-analysis was based on secondary study-level data, and the evaluation indicators varied greatly among different RCTs. Low quality of RCTs ( 2 out of 21 RCTs scored less than 4 by the modified Jadad scale) might influence the pooled results. Unlike one meta-analysis report recently published with only one RCT included [47], we only included RCTs $(n=21)$. Second, few studies reported the disease recurrence and overall survival data and the like. For them, it was difficult to adequately measure the long-term efficacy of NOSE surgery. Third, of the 21 included RCTs, one was reported in Belgium, one was in Hong Kong, China, and the others were all reported in mainland China. The enrolled studies were not widely distributed all over the world, which would limit the study finding to extrapolate further. Last, different operation skills and study population might induce potential bias among the included RCTs. Therefore, a large-sized wellcontrolled RCT is warranted to further verify the advantages and disadvantages of NOSES after following a uniform surgery guideline.

\section{Conclusion}

This report appeared the first comprehensive meta-analysis to quantitatively synthesize data from RCTs of laparoscopic resection with NOSE versus conventional laparoscopy. Compared with CL, NOSE surgery demonstrated multiple advantages in terms of shorter hospital stay after surgery, less pain, faster recovery from surgery, better cosmetic results, and most importantly, fewer postoperative complications. Even so, well-controlled RCTs of the NOSES following a uniform surgery guideline are warranted in the future.

\section{Abbreviations}

CRC: $\quad$ Colorectal cancer

CI: $\quad$ Confidence interval

CL: $\quad$ Conventional laparoscopy

ERAS: $\quad$ Enhanced recovery after surgery

MD: $\quad$ Mean difference

NA: $\quad$ Not applicable

NOSE: Natural orifice specimen extraction

NOSES: Natural orifice specimen extraction surgery

NOTES: Natural orifice transluminal endoscopic surgery 
PRISMA: Preferred reporting items for systematic reviews and meta-analyses

RCTs: Randomized controlled trials

RR: $\quad$ Rate ratio.

\section{Data Availability}

The data supporting this meta-analysis are from previously reported studies and datasets, which have been cited.

\section{Disclosure}

The funders had no role in study design, conduct, data collection and analysis, or paper preparation and submission of the study.

\section{Conflicts of Interest}

All authors have no conflicts of interest or financial ties to disclose.

\section{Authors' Contributions}

C.F and Y.Y participated in the preparation of study concept and design and performed quality assessment. Z.Z, L.C, and J.L performed study selection, data extraction, and quality assessment and drafted the manuscript. F.J, Y.S, and X.Y carried out the data statistical analysis and interpretation and also made major revisions to the manuscript. All of the authors have read and approved the final version of the manuscript. Zhuqing Zhou, Lin Chen, Jie Liu, and Fang Ji contributed equally to this work and should be considered co-first authors.

\section{Acknowledgments}

This work was supported by the Shanghai Health Commission Clinical Research Project (202040303), the National Natural Science Foundation of China (No. 81573004 and No. 81773275), the Top-level Clinical Discipline Project of Shanghai Pudong (No. PWYgf2018-04), and the Pudong New District Health and Family Planning Commission Youth Science and Technology Project (No. PW2016B-4). The authors would like to thank Dr. Sam Zhong for his kind support with data analyses in the present study.

\section{References}

[1] R. Abdel-Samad, P. Aouad, and N. Darwiche, "Natural and synthetic retinoids in preclinical colorectal cancer models," Anti-Cancer Drugs, vol. 30, no. 7, article e0802, 2019.

[2] A. Ishibe, M. Ota, S. Fujii et al., "Midterm follow-up of a randomized trial of open surgery versus laparoscopic surgery in elderly patients with colorectal cancer," Surgical Endoscopy, vol. 31, no. 10, pp. 3890-3897, 2017.

[3] W. Xishan and Alliance CN, "Expert consensus of natural orifice specimen extraction surgery in colorectal neoplasm (2017 edition)," Chinese Journal of Colorectal Diseases, vol. 6, no. 4, pp. 266-272, 2017.
[4] A. M. Wolthuis, A. D. Van Overstraeten, and A. D'Hoore, "Laparoscopic natural orifice specimen extraction-colectomy: a systematic review," World Journal of Gastroenterology, vol. 20, no. 36, pp. 12981-12992, 2014.

[5] F. A. Costantino, M. Diana, J. Wall, J. Leroy, D. Mutter, and J. Marescaux, "Prospective evaluation of peritoneal fluid contamination following transabdominal vs. transanal specimen extraction in laparoscopic left-sided colorectal resections," Surgical Endoscopy, vol. 26, no. 6, pp. 1495-1500, 2012.

[6] B. Ma, X. Z. Huang, P. Gao et al., "Laparoscopic resection with natural orifice specimen extraction versus conventional laparoscopy for colorectal disease: a meta-analysis," International Journal of Colorectal Disease, vol. 30, no. 11, pp. 1479-1488, 2015.

[7] D. Moher, A. Liberati, J. Tetzlaff, D. G. Altman, and The PRISMA Group, "Preferred reporting items for systematic reviews and meta-analyses: the PRISMA statement," PLoS Medicine, vol. 6, no. 7, article e1000097, 2009.

[8] J. G. Wright, M. F. Swiontkowski, and J. D. Heckman, "Introducing levels of evidence to the journal," The Journal of Bone and Joint Surgery. American Volume, vol. 85, no. 1, pp. 1-3, 2003.

[9] Oxford Centre for Evidence-based Medicine - Levels of Evidence, 2009, http://www.cebm.net/oxford-centre-evidencebased-medicine-levels-evidence-march-2009/.

[10] S. A. Olivo, L. G. Macedo, I. C. Gadotti, J. Fuentes, T. Stanton, and D. J. Magee, "Scales to assess the quality of randomized controlled trials: a systematic review," Physical Therapy, vol. 88, no. 2, pp. 156-175, 2008.

[11] T. Greenhalgh, "Assessing the methodological quality of published papers,” BMJ, vol. 315, no. 7103, pp. 305-308, 1997.

[12] M. Oremus, C. Wolfson, A. Perrault, L. Demers, F. Momoli, and Y. Moride, "Interrater reliability of the modified Jadad quality scale for systematic reviews of Alzheimer's disease drug trials," Dementia and Geriatric Cognitive Disorders, vol. 12, no. 3, pp. 232-236, 2001.

[13] A. Hoyer, Metaanalysis with R. G.Schwarzer, J. R.Carpenter, G.Rücker vol. 59, Springer, Berlin, DE, 2015.

[14] S. P. Hozo, B. Djulbegovic, and I. Hozo, "Estimating the mean and variance from the median, range, and the size of a sample," BMC Medical Research Methodology, vol. 5, no. 1, 2005.

[15] J. L. Peters, A. J. Sutton, D. R. Jones, K. R. Abrams, and L. Rushton, "Comparison of two methods to detect publication bias in meta-analysis," JAMA, vol. 295, no. 6, pp. 676-680, 2006.

[16] D. Jackson, I. R. White, and R. D. Riley, "Quantifying the impact of between-study heterogeneity in multivariate metaanalyses," Statistics in Medicine, vol. 31, no. 29, pp. 38053820, 2012.

[17] Y. Ding, Z. Li, H. Gao, Y. Cao, and W. Jin, "Comparison of efficacy between natural orifice specimen extraction without abdominal incision and conventional laparoscopic surgery in the treatment of sigmoid colon cancer and upper rectal cancer," Journal of BUON, vol. 24, no. 5, pp. 1817-1823, 2019.

[18] A. M. Wolthuis, S. Fieuws, A. van den Bosch, A. de Buck van Overstraeten, and A. D'Hoore, "Randomized clinical trial of laparoscopic colectomy with or without natural-orifice specimen extraction," The British Journal of Surgery, vol. 102, no. 6, pp. 630-637, 2015.

[19] Z. Zhu, K. J. Wang, G. R. Orangio et al., "Clinical efficacy and quality of life after transrectal natural orifice specimen 
extraction for the treatment of middle and upper rectal cancer," Journal of Gastrointestinal Oncology, vol. 11, no. 2, pp. 260-268, 2020.

[20] A. L. Leung, H. Y. S. Cheung, B. K. L. Fok, C. C. C. Chung, M. K. W. Li, and C. N. Tang, "Prospective randomized trial of hybrid NOTES colectomy versus conventional laparoscopic colectomy for left-sided colonic tumors," World Journal of Surgery, vol. 37, no. 11, pp. 2678-2682, 2013.

[21] M. Shen and X. Yang, "Clinical efficacy and quality of life with natural orifice specimen extraction (NOSES) surgery for rectal cancer," Journal of Colorectal \& Anal Surgery, vol. 25, no. 4, pp. 441-445, 2019.

[22] H. Gao, "Compare the effect of laparoscopic colorectal cancer specimens with natural cavity removal (NOSE) and conventional laparoscopic radical colorectal cancer (LCR) on postoperative recovery," China Medical Device Information, vol. 26, no. 4, pp. 28-29, 2020.

[23] Q. Zhao, J. Guo, and Q. Cui, "Experiences of total laparoscopic resection for rectal cancer by NOSES," Chinese Journal of Current Advances in General Surgery, vol. 22, 2019.

[24] D. Feng, "Effect on postoperative rehabilitation of natural orifice specimen extraction for laparoscopic radical surgery and conventional laparoscopic colorectal resection," Modern Oncology, vol. 26, no. 22, pp. 3601-3605, 2018.

[25] J. Wang, "Perioperative nursing of rectal cancer treated by natural orifice specimen extraction surgery under the concept of accelerated rehabilitation surgery," Electronic Journal of Clinical Medical Literature, vol. 6, no. 79, 2019.

[26] H. Ding, "Effects on oxidative stress reaction immune function and inflammation during NOSE radical surgery for colorectal cancer," Chinese Journal of Modern Operative Surgery, vol. 21, no. 1, 2017.

[27] K. Wang, "Short-term clinical efficacy and quality of life in patients with colorectal cancer after natural orifice specimen extraction surgery," Journal Of Tongji University, vol. 40, no. 6, pp. 789-794, 2019.

[28] D. Zhao, "Effect of total laparoscopic resection for colorectal cancer by NOSE," China Journal of Endoscopy, vol. 23, no. 12 , pp. 36-40, 2017.

[29] L. Zhao and Z. Liu, "Complete laparoscopic radical resection of colorectal cancer by natural orifice specimen extraction," Renowned Doctor, vol. 4, p. 36, 2019.

[30] X. Li, "Clinical study on the treatment of sigmoid colon and rectal cancer by transluminal resection combined with accelerated rehabilitation surgery," Modern Hospitals, vol. 18, no. 10, pp. 1551-1554, 2018.

[31] M. Zhang and Y. Wu, "Influence of total laparoscopic resection for rectal cancer by natural orifice specimen extraction without abdominal auxiliary incision on the third space effect," Chinese Journal of Surgical Oncology, vol. 12, no. 1, pp. 54-59, 2020.

[32] B. Liu, B. Liu, C. Bao, B. Xu, B. Shen, and Z. Yang, “Application of terminal ileum suspension combined with natural orifice specimen extraction in anus-preserving surgery for low rectal cancer," The Journal of Practical Medicine, vol. 35, no. 22, pp. 3508-3512, 2019.

[33] Z. Huang, "Clinical value of total laparoscopic non abdominal auxiliary incision in sigmoid and high rectal cancer," Chinese Journal of Practical Medicine, vol. 45, no. 14, 2018.

[34] Z. Hu, W. Li, C. Su, and W. Wei, "Clinical application value of completelaparoscopic rectal valgus and rectal cancer with no auxiliary incision," Chinese Journal Surgery Oncologia, vol. 11, 2019.

[35] Y. Yue and L. Zhu, "Effect of NOSES VIsurgery in sigmoid colon cancer: a study of 40 patients," Journal of Colorectal \& Anal Surgery, vol. 24, no. 5, pp. 468-472, 2018.

[36] L. Ma, X. Zhang, F. Wang, and A. Wu, "Clinical observation of rectal carcinoma treated by complete laparoscopic total mesorectal resection without auxiliary incision," Modern Digestion \& Intervention, vol. 24, 2019.

[37] Y. Chen, R. Guo, J. Xie, Z. Liu, P. Shi, and Q. Ming, "Laparoscopy combined with transanal endoscopic microsurgery for rectal cancer: a prospective randomized controlled study," Chinese Journal of Minimally Invasive Surgery, vol. 14, no. 9, pp. 775-778, 2014.

[38] J. Yu, Comparative Study of Postoperative Outcomes of NOSES and Conventional Laparoscopic Radical Resection of Rectal Cancer, Qingdao University, 2019.

[39] H. Zhang, Application of NOSE surgery combined with rapid rehabilitation in the treatment of colorectal cancer, Southern Medical University, 2017.

[40] $\mathrm{H}$. Chen, The application of rapid rehabilitation surgery in laparoscopic natural orifce specimen extraction surgery, Henan University, 2018.

[41] Z. Chen, Prospective randomized controlled trial of laparoscopic-assisted radical left colorectomy transanal specimen extraction using the Cai Tube, Fujian Medical University, 2018.

[42] Z. Q. Zhou, K. Wang, T. du et al., “Transrectal natural orifice specimen extraction (NOSE) with oncological safety: a prospective and randomized trial," The Journal of Surgical Research, vol. 254, pp. 16-22, 2020.

[43] A. O. Rasulov, Z. Z. Mamedli, K. E. Dzhumabaev, V. M. Kulushev, and N. A. Kozlov, "Total mesorectal excision in rectal cancer management: laparoscopic or transanal?," Khirurgiia (Mosk), vol. 5, no. 5, pp. 37-44, 2016.

[44] X. Guan, Z. Liu, A. Parvaiz et al., "International consensus on natural orifice specimen extraction surgery (NOSES) for gastric cancer (2019)," Gastroenterology Report, vol. 8, no. 1, pp. 5-10, 2020.

[45] M. A. Yagci, C. Kayaalp, and N. H. Novruzov, "Intracorporeal mesenteric division of the colon can make the specimen more suitable for natural orifice extraction," Journal of Laparoendoscopic \& Advanced Surgical Techniques. Part A, vol. 24, no. 7, pp. 484-486, 2014.

[46] N. O. S. E. S. Alliance, "Professional Committee of Natural Orifice Specimen Extraction Surgery, C.C.C.o.C.M.D.A., expert consensus of natural orifice specimen extraction surgery in colorectal neoplasm (2019)," Chinese Journal Colorectal Disease, vol. 8, no. 4, pp. 336-342, 2019.

[47] J. Lin, S. Lin, Z. Chen et al., "Meta-analysis of natural orifice specimen extraction versus conventional laparoscopy for colorectal cancer," Langenbeck's Archives of Surgery, vol. 406, no. 2, pp. 283-299, 2021. 